\title{
Cuidados para el manejo de delirio en Unidades de Paciente Crítico: una revisión integrativa $^{*}$
}

\section{Care management of delirium in Critical Patient Units: an integrative review}

\section{Cuidados para o manejo de delirium em Unidades de Pacientes Críticos: uma revisão integrativa}

\section{Gabriela Flores-Oñate ${ }^{1}$; Paula Ceballos-Vásquez² \& Rocio Mejías-Parada ${ }^{3}$}

${ }^{1}$ Enfermera, Especialista en Cuidados Críticos del Adulto. Hospital Base Curicó, Chile. Orcid: 0000-00022198-8252. Correo electrónico: eu.gabrielaflores@gmail.com

${ }^{2}$ Enfermera. Doctor en Enfermería. Departamento de Enfermería. Universidad Católica del Maule, Talca, Chile. Orcid: 0000-0002-3804-5146.Correo electrónico: pceballos@ucm.cl.

${ }^{3}$ Enfermera, Especialista en Cuidados Críticos del Adulto. Hospital Regional de Talca, Chile. Correo electrónico: rocio_mejias_@hotmail.com

Correspondencia: Paula Ceballos-Vásquez. Avenida San Miguel \#3505 C.P: 3480112.

Correo electrónico de contacto: pceballos@ucm.cl.

Cómo citar este artículo: Flores-Oñate, G., Ceballos-Vásquez, P.,\& Mejías-Parada, R. (2021). Cuidados para el manejo de delirio en Unidades de Paciente Crítico: una revisión integrativa. Cultura de los Cuidados (Edición digital), 25(59). Recuperado de http://dx.doi.org/10.14198/cuid.2021.59.15

Recibido:02/11/2020 Aceptado: 12/01/2021

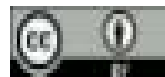

\footnotetext{
* Trabajo que emerge del Módulo de Análisis Crítico Bibliográfico en el marco del Programa de Especialización en Cuidados Críticos del Adulto
} perteneciente a la Facultad de Ciencias de la Salud de la Universidad Católica del Maule. 


\section{RESUMEN}

Objetivo: analizar en la evidencia bibliográfica los aspectos que influyen en el desarrollo del delirio, manejo, evaluación objetiva para su pesquisa y los cuidados relacionados a la promoción y prevención de éste en los usuarios hospitalizados en unidades críticas. Metodología: Se realizó una revisión integrativa, en bases de datos CINAHL; SCOPUS y Scielo. Fueron seleccionados 19 artículos limitados por año (2016-2017). Resultado: Se identificaron cuatro factores que impactan en el delirio: la fisiopatología/factores de riesgo, la prevención y promoción, la evaluación objetiva y el manejo en unidades críticas. Conclusión: La Gestión del Cuidado se encuentra a cargo de Enfermería, por ende, es fundamental no dejar esta temática de lado debido a que su incidencia aumentará los días de hospitalización, días de ventilación mecánica y mortalidad, impactando negativamente en la calidad de los cuidados.

Palabras clave: Delirio, enfermería; cuidados críticos; unidades de cuidados intensivos.

\section{ABSTRACT}

Aim: To identify aspects that influence the development of delirium, its management, evaluation and care in relation to the promotion and the prevention of delirium. Methodology: An integrative review was carried out in the following databases; CINHAL, SCOPUS and Scielo. 19 articles were selected, limited by year (2016-2017). Result: Four factors that have an impact on delirium were identified: pathophysiology / risk factors, prevention and promotion, objective evaluation and management in critical units. Conclusion: Nursing is in charge of care management, therefore, it is essential not to leave this issue aside because its incidence will increase the days of hospitalization, days of mechanical ventilation and mortality, negatively impacting the quality of care.

Keywords: Delirium; nursing; critical Care; intensive care units

\section{RESUMO}

Objetivo: Identificar aspectos que influenciam o desenvolvimento do delirium, seu manejo, avaliação objetiva para sua pesquisa e cuidados relacionados à promoção e prevenção deste. Metodologia: Uma revisão integrativa foi realizada nas bases de dados da CINAHL; SCOPUS e Scielo. Foram selccionados 19 artigos limitados por ano (2016-2017). Resultado: Foram identificados quatro fatores que têm impacto no delirium: fisiopatologia / fatores de risco, prevenção e promoção, avaliação objetiva e manejo em unidades críticas. Conclusão: A gerência do cuidado é responsável pela enfermagem, portanto, é imprescindível não deixar de lado essa questão, pois sua incidência aumentará os dias de internação, dias de ventilação mecânica e mortalidade, impactando negativamente na qualidade do cuidado.

Palavras-chave: Delirium; enfermagem; cuidados críticos; unidades de terapia intensiva.

\section{INTRODUCCIÓN}

El delirio se manifiesta como un cambio agudo y fluctuante del estado mental, caracterizado por nivel alterado de conciencia o un pensamiento desorganizado, $\mathrm{y}$ es una forma frecuente $\mathrm{y}$ grave de disfunción cerebral que se produce en los pacientes críticos (Barr et al., 2013; Salluh et al., 2015). El delirio se puede manifestar de diferentes formas, $\mathrm{y}$, por ende, se ha clasificado en 3 subtipos: la persona con 
Revista científica de la Asociación de Historia y Antropología de los Cuidados (Universidad de Alicante)

delirio hiperactivo puede presentar agitación, inquietud, conductas agresivas con el entorno y consigo mismo, al punto tener caídas, auto-extubaciones, autoretiro de vías, sondas y catéteres, etc. Mientras que en el delirio hipoactivo, la persona suele estar somnolienta, inatento, con menos cambios de humor y menos alteraciones del ciclo sueño-vigilia, por ello, suele pasar desapercibido debido a su presentación tenue y letárgica. Por último, se produce un delirio de tipo mixto, el cual tiene manifestaciones de tipo hipo e hiperactividad de manera secuencial (Palencia et al., 2008). Estudios demuestran que el delirio hiperactivo se presenta entre un 1 a 23\%, el hipoactivo entre un 19 y 68\% y el mixto entre un 14 y 55\%. Autores señalan que está asociado a aumento de la mortalidad, duración de la ventilación mecánica, estadía prolongada en Unidades Criticas (UCI), y problemas de salud mental a largo plazo (Van de Boogaard et al., 2012; Reade et al., 2014), por lo tanto, representa un desafío para los profesionales que se desempeñan en UCI, y un problema de salud pública (Bui et al., 2017). En la actualidad, no se reconoce con exactitud la causa de este trastorno (Ely et al., 2004), sin embargo, se ha logrado evaluar, clasificar y manejar cuando existe un equipo de salud competente (Salazar et al., 2012). Por ello, emerge la pregunta ¿Un cuidado competente, podría aportar al manejo integral del delirio? El objetivo de esta revisión integrativa fue analizar en la evidencia bibliográfica los aspectos que influyen en el desarrollo del delirio, manejo, evaluación objetiva para su pesquisa y los cuidados relacionados a la promoción y prevención de éste en los usuarios hospitalizados en unidades críticas. Y así, entregar directrices concretas al equipo de salud que se desempeña en UCI, respecto al delirio en pacientes críticos.

\section{PROCEDIMIENTOS} METODOLÓGICOS DE LA REVISIÓN INTEGRADORA

Se realizó búsqueda de literatura científica sobre delirio en Unidad de Cuidados Intensivos (UCI), en las bases de datos: Scopus, CINAHL y Scielo, para ello, se utilizaron los descriptores "Delirium” e "Intensive Care Unit" y como operador booleano el conector AND. Se obtuvieron primeramente 4500 artículos, los cuales se redujeron a 95 según los criterios de limitación; año (2016-2017), texto completo, publicaciones académicas, idioma (ingles-portugués-español) y edad (mayores de 18 años), los cuales se redujeron a 23, tras excluir por relevancia en el título, resumen y palabras clases. Finalmente, tras realizar una revisión de los artículos y de extracción de datos, se incluyeron 19 artículos, todo lo señalado se resume en la figura $\mathrm{N}^{\circ} 1$. Como criterios de inclusión se indican: estudios realizados en personas mayores de 18 años, hospitalizados en Unidades Críticas (UCI y urgencia) y tener incorporado en el título, resumen y/o palabras claves, los conceptos de delirio y/o Unidad de Cuidados Intensivos. Los criterios de exclusión fueron: estudios en personas con trastornos neurológicos, estudios exclusivos de una patología o en el uso de un medicamento en específico. En la tabla 1 se describen los artículos seleccionados según autor, objetivo, método, muestra y aportes para la revisión 
Articulos identificados en base de datos electidnicos: $(n=4.500)$

QNAHL $1250 ;$ SCOPUS= $3239 ;$ SCELD $=11$

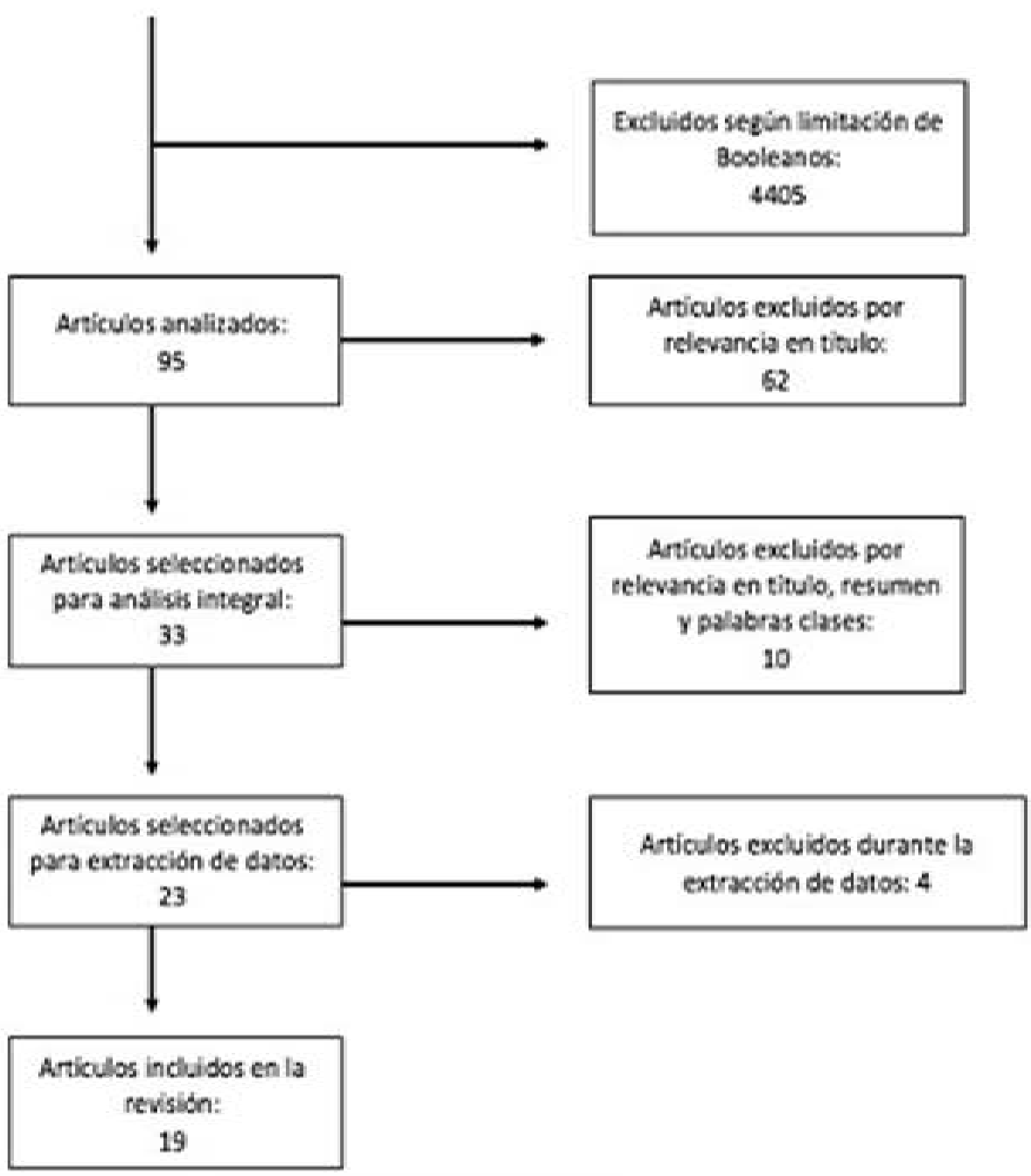

Figuro 1. Proceso de selección de estud os- PRISvA dagram flow Fuente: Elaboración Propla 
Tabla 1. Artículos seleccionados para el análisis

\begin{tabular}{|c|c|c|c|}
\hline $\begin{array}{ll}\text { Identificación } & \text { del } \\
\text { Estudio } & \end{array}$ & Objetivo & Método y Muestra & Aportes para la Revisión \\
\hline \multirow[t]{2}{*}{$\begin{array}{l}\text { E1 Pereira et al., } \\
\text { (2016) }\end{array}$} & $\begin{array}{l}\text { Identificar los factores de riesgos } \\
\text { modificables por enfermeros, asociados } \\
\text { con el desarrollo del delirio en } \\
\text { hospitalizados en una UCI intensivos }\end{array}$ & $\begin{array}{l}\text { Exploratorio- } \\
\text { descriptivo. }\end{array}$ & \multirow{2}{*}{$\begin{array}{l}\text { Se identificaron factores de riesgo modificables en } \\
\text { el desarrollo del delirio, estos se relacionan con } \\
\text { alimentación, hidratación, gestión de dispositivos } \\
\text { clínicos, promoción de la visita. oxigenoterapia } \\
\text { adecuada, etc. Es relevante posicionar a } \\
\text { enfermeros, por sobre todo a los especialistas, en la } \\
\text { gestión de riesgo creando y manteniendo ambientes } \\
\text { terapéuticos más seguros. }\end{array}$} \\
\hline & nivel II de un hospital central & $\begin{array}{l}\text { hospitalizados y } \\
\text { seleccionados de } \\
\text { forma intencional. }\end{array}$ & \\
\hline
\end{tabular}

E2 Ditrich et al., Determinar la incidencia y los factores (2016) predictivos del delirio de nueva aparición y su impacto en el resultado en pacientes adultos críticamente enfermos

\begin{abstract}
Cohorte Prospectivo
240 usuarios

La incidencia de delirio en personas con infecciones del torrente sanguíneo es alta (60\% de la muestra evaluada). Los usuarios delirantes tuvieron una mortalidad de casi el doble y las probabilidades de tener delirio aumentaron a mayor número de catéteres y drenajes. Se utilizó como predictor, el puntaje Intensive Care Delirium Screening Checklist (ICDSC.)
\end{abstract}

E3 Kim et al., Desarrollar puntuación de predicción (2016) del delirio que permita la identificación de individuos con alta probabilidad de delirio postoperatorio.

\section{Prospectivo \\ Observacional}

561 usuarios
Se identificaron aquellos usuarios con mayor predisposición a desarrollar delirio. Por medio de un Delphi, se determinaron los posibles factores de riesgo para el derilio post operatorio. Se puntualiza la importancia de enfermería, utilizando la escala Nursing delirium screening checklist (Nu-DESC.)
E4 Morandi et al., Evaluar el (ABCDEF) Bundle para (2017)

\section{implementar las guías de Dolor,} Agitación y Delirio.
Encuesta transversal en línea

1521 encuestados de 47 países
El (ABCDEF) Bundle aborda una práctica objetiva en la UCI que se asocia finalmente a la seguridad del paciente. Integra la evaluación, prevención y manejo del dolor, despertar y respirar espontáneas, analgesia, sedación, delirio, movilidad y participación de la familia.
E5 Goulart et al., Evaluar efecto de un modelo de visita (2017) prolongada comparado con un modelo de visita restringida sobre ocurrencia de delirio
Estudio Prospectivo de un antes $y$ un después en un solo centro

286 usuarios de UCI
Los usuarios con modelos de visita extendida (12 horas) tuvieron una duración más corta de delirio/coma, además, disminuyó el estrés, la ansiedad y se optimizó la orientación a diferencia de aquellos usuarios que presentaron un modelo de visita restringida (4,5 horas).
E6 Sullinger et al., Evaluar impacto de implementar un (2016) protocolo de tratamiento de delirio v/s cantidad de días sin delirio experimentado por usuarios.
Observacional Prospectivo

593 usuarios Críticos
La implementación de un protocolo de delirio con intervenciones farmacológicas y no farmacológicas redujo significativamente los días delirantes y la duración de estadía en UCI. Además, se demostró que las intervenciones con el Bundle ABCDEF son beneficiosas en el paciente crítico. 


\begin{tabular}{|c|c|c|c|}
\hline $\begin{array}{l}\text { E7 Wood et al., } \\
\text { (2017) }\end{array}$ & $\begin{array}{l}\text { Comprobar si la oxigenación } \\
\text { deficiente del tejido cerebral } \\
\text { (BtO2) durante las primeras } 24 \\
\text { horas de enfermedad crítica se } \\
\text { correlaciona con la proporción de } \\
\text { tiempo de delirio }\end{array}$ & $\begin{array}{l}\text { Observacional } \\
\text { Prospectivo } \\
88 \text { usuarios }\end{array}$ & $\begin{array}{l}\text { La oxigenación cerebral deficiente durante } \\
\text { las primeras } 24 \text { horas de una enfermedad } \\
\text { crítica contribuye al desarrollo del delirio. }\end{array}$ \\
\hline $\begin{array}{l}\text { E8 Bui et al., } \\
\text { (2017) }\end{array}$ & $\begin{array}{l}\text { Comparar proporciones de } \\
\text { usuarios con delirio detectados por } \\
\text { CAM-ICU que recibieron la } \\
\text { documentación administrativa para } \\
\text { el delirio utilizando la clasificación } \\
\text { ICD-9-CM. }\end{array}$ & $\begin{array}{l}\text { Cohorte- } \\
\text { retrospectivo } \\
1055 \text { usuarios en } \\
\text { UCI quirúrgica }\end{array}$ & $\begin{array}{l}\text { La clasificación internacional de } \\
\text { enfermedades (ICD), en la novena revisión } \\
\text { (ICD-9-CM) solo presentó } 32 \text { códigos } \\
\text { implícitos y explícitos para identificar el } \\
\text { delirio. La ICD-9-CM en comparación con el } \\
\text { CAM-ICU presenta estimaciones sesgadas y } \\
\text { poca sensibilidad, por lo que subestimaría la } \\
\text { prevalencia del delirio. Además fue más } \\
\text { propenso a identificar delirio } \\
\text { hiperactivo/mixto que el hipoactivo. }\end{array}$ \\
\hline $\begin{array}{l}\text { E9 Wolters et al., } \\
\text { (2016) }\end{array}$ & $\begin{array}{l}\text { Determinar si el delirio durante la } \\
\text { estancia en la UCI se asocia con } \\
\text { problemas de salud mental a largo } \\
\text { plazo }\end{array}$ & $\begin{array}{l}\text { Cohorte- } \\
\text { Prospectivo } \\
\text { Encuesta a } 567 \\
\text { usuarios evaluados } \\
1 \text { año después del } \\
\text { alta de una UCI } \\
\text { Médico- } \\
\text { Quirúrgica }\end{array}$ & $\begin{array}{l}\text { La aparición del delirio durante la estancia en } \\
\text { UCI no aumentó el riesgo de estos problemas } \\
\text { de salud mental a largo plazo. Se utilizó como } \\
\text { herramienta el "algoritmo de } 5 \text { pasos para la } \\
\text { clasificación del estatus mental", que incluye } \\
\text { la escala de agitación de Richmond, CAM- } \\
\text { ICU, entre otras evaluaciones. }\end{array}$ \\
\hline $\begin{array}{l}\text { E10 Xing et al., } \\
\text { (2017) }\end{array}$ & $\begin{array}{l}\text { Evaluar el conocimiento, actitudes } \\
\text { y manejo respecto al delirio de } \\
\text { médicos y enfermeras de UCI, y } \\
\text { evaluar las barreras percibidas } \\
\text { relacionadas con la monitorización } \\
\text { del delirio en la UCI en China. }\end{array}$ & $\begin{array}{l}\text { Encuesta } \\
\text { descriptiva con } \\
\text { cuestionario de } \\
\text { autoinforme a } \\
1556 \text { enfermeros y } \\
\text { médicos (2:1) de } \\
74 \text { hospitales en } \\
\text { total. }\end{array}$ & $\begin{array}{l}\text { Se reconoce la necesidad crítica de educar a } \\
\text { enfermeros y médicos sobre evaluación } \\
\text { rutinaria y tratamiento oportuno del delirio en } \\
\text { la UCI. Si bien es evaluado por el } 81 \% \text { del } \\
\text { personal ( } 56,6 \% \text { por médicos y } 18,65 \% \text { por } \\
\text { enfermeros), solo el } 31,6 \% \text { utilizó una } \\
\text { herramienta de fiabilidad y validez como el } \\
\text { CAM-ICU o la ICDSC. Además solo el } \\
17,7 \% \text { realizó la evaluación de la sedación } \\
\text { como parte fundamental del delirio. }\end{array}$ \\
\hline $\begin{array}{l}\text { E11 Da Silba et } \\
\text { al., (2017) }\end{array}$ & $\begin{array}{l}\text { Contar la experiencia de formación } \\
\text { de enfermeras para la } \\
\text { implementación de la evaluación } \\
\text { sistemática de delirio por el CAM- } \\
\text { ICU }\end{array}$ & $\begin{array}{l}\text { Relato de } \\
\text { experiencia } \\
\text { Se instrumentalizó } \\
\text { a } 60 \text { enfermeros de } \\
\text { una UCI de un } \\
\text { hospital de tercer } \\
\text { nivel en Sao Paulo }\end{array}$ & $\begin{array}{l}\text { El proceso de implementación de evaluación } \\
\text { de CAM-ICU se compuso de } 4 \text { etapas: 1) } \\
\text { Análisis de situación; 2) Planificación; 3) } \\
\text { Formación del personal: } 15 \text { días en fase } \\
\text { teórica y } 15 \text { días más en entrenamiento } \\
\text { práctico; y 4) Evaluación aplicación del } \\
\text { instrumento. La experiencia mostró aumento } \\
\text { de pacientes diagnosticados, como también } \\
\text { dificultad para aplicar la escala de sedación y } \\
\text { agitación de Richmond. }\end{array}$ \\
\hline $\begin{array}{l}\text { E12 Green et al., } \\
\text { (2017) }\end{array}$ & $\begin{array}{l}\text { Validar la EDTB-ICU (Edinburgh } \\
\text { Delirium Test Box-ICU) para } \\
\text { detectar y cuantificar la falta de } \\
\text { atención en pacientes con delirio. }\end{array}$ & $\begin{array}{l}\text { Estudio } \\
\text { exploratorio } \\
\text { Prospectivo de } \\
\text { casos y controles } \\
\text { de } 20 \text { usuarios }\end{array}$ & $\begin{array}{l}\text { La herramienta de evaluación Edinburgh } \\
\text { Delirium Test Box-ICU (EDTB-UCI) } \\
\text { proporciona un método fácil de aprender, es } \\
\text { objetivo, rápido ( } 3-7 \text { minutos), sensible y } \\
\text { específico para detectar el delirio en UCI. La } \\
\text { evaluación es adecuada para su uso en } \\
\text { pacientes verbales y no verbales (Intubados). } \\
\text { Destaca la participación de enfermería en el } \\
\text { estudio. }\end{array}$ \\
\hline $\begin{array}{l}\text { E13 Mori et al., } \\
\text { (2016) }\end{array}$ & $\begin{array}{l}\text { Identificar incidencia de delirio, } \\
\text { comparar los datos demográficos y } \\
\text { clínicos de los pacientes con y sin } \\
\text { delirio, y verificar los factores } \\
\text { relacionados con el delirio en } \\
\text { pacientes críticos. }\end{array}$ & $\begin{array}{l}\text { Cohorte } \\
\text { prospectiva } \\
149 \text { usuarios } \\
\text { hospitalizado en la } \\
\text { UCI de un hospital } \\
\text { universitario. }\end{array}$ & $\begin{array}{l}\text { Los factores relacionados con delirio fueron; } \\
\text { edad avanzada, uso de sedantes y } \\
\text { analgésicos. El cuidado de enfermería es } \\
\text { relevante para prevenir delirio a través de } \\
\text { medidas no farmacológicas principalmente } \\
\text { en pacientes ancianos. }\end{array}$ \\
\hline
\end{tabular}




\begin{tabular}{|c|c|c|c|}
\hline $\begin{array}{l}\text { E14 Khan et al., } \\
\text { (2017) }\end{array}$ & $\begin{array}{l}\text { Evaluar la fiabilidad y la validez de } \\
\text { una herramienta de severidad del } \\
\text { delirio de la UCI el Método de } \\
\text { evaluación de confusiones para la } \\
\text { escala de gravedad del delirio ICU- } \\
7 .\end{array}$ & $\begin{array}{l}\text { Cohorte } \\
\text { observacional } \\
518 \text { pacientes de } \\
\text { UCI médicas, } \\
\text { quirúrgicas y } \\
\text { progresivas de tres } \\
\text { hospitales } \\
\text { académicos }\end{array}$ & $\begin{array}{l}\text { La escala de gravedad del delirio CAM-ICU- } \\
7 \text { es válida, confiable, y la medida práctica de } \\
\text { la severidad del delirio entre los pacientes de } \\
\text { la UCI. A diferencia del DRS-R-98, por su } \\
\text { uso limitado en UCI, debido a la cantidad de } \\
\text { tiempo requerido para la aplicación, } \\
\text { requisitos de entrenamiento extensos y los } \\
\text { factores clínicos específicos de la UCI, } \\
\text { incluida gravedad de la enfermedad y } \\
\text { ventilación mecánica. }\end{array}$ \\
\hline $\begin{array}{l}\text { E15 Yang et al., } \\
\text { (2017) }\end{array}$ & $\begin{array}{l}\text { Evaluar los factores de riesgo para } \\
\text { el delirio en pacientes con sedación } \\
\text { secuencial. }\end{array}$ & $\begin{array}{l}\text { Estudio } \\
\text { planificado, } \\
\text { pacientes } \\
\text { seleccionados de } \\
\text { un estudio previo } \\
141 \text { pacientes }\end{array}$ & $\begin{array}{l}\text { Los factores de riesgo significativos son: } \\
\text { edad avanzada }(\geq 51 \text { años }) \text {, fumadores, } \\
\text { puntaje SOFA }(\geq 14) \text { y dosis de } \\
\text { mantenimiento de midazolam con fentanilo. } \\
\text { Al utilizar sedación secuencial, con } \\
\text { dexmedetomidina se demostró menor riesgo } \\
\text { de delirio comparado con el uso de } \\
\text { midazolam. }\end{array}$ \\
\hline $\begin{array}{l}\text { E16 Boettger et } \\
\text { al., (2017) }\end{array}$ & $\begin{array}{l}\text { Evaluar impacto de sedación sobre } \\
\text { la fenomenología del delirio } \\
\text { medido por DRS-R-98 y el } \\
\text { diagnóstico de delirio determinado } \\
\text { por DSM-IV-TR. }\end{array}$ & $\begin{array}{l}\text { Estudio } \\
\text { prospectivo } \\
\text { cohortes } \\
225 \text { usuarios }\end{array}$ & $\begin{array}{l}\text { La somnolencia aumenta ocho veces las } \\
\text { probabilidades de desarrollar un delirio más } \\
\text { severo. Además, ésta por sí misma causa } \\
\text { deterioro atencional, alteraciones en el ciclo } \\
\text { sueño-vigilia y anomalías del lenguaje, por lo } \\
\text { tanto, su perturbación en la conciencia } \\
\text { aumenta el delirio. }\end{array}$ \\
\hline $\begin{array}{l}\text { E17 Garpestad, } \\
\text { y Devlin, (2017) }\end{array}$ & $\begin{array}{l}\text { Evaluar la relación causal entre la } \\
\text { polifarmacia y el delirio en estado } \\
\text { crítico, adultos mayores. }\end{array}$ & $\begin{array}{l}\text { Análisis } \\
\text { cohorte }\end{array}$ & $\begin{array}{l}\text { La polifarmacia es común en los adultos } \\
\text { mayores (AM) en estado crítico. Se produce } \\
\text { delirio en más del } 50 \% \text { de los AM ingresados } \\
\text { en la UCI por la administración de } \\
\text { medicamentos usados en forma rutinaria } \\
\text { (anti-psicóticos). Se deben implementar } \\
\text { intervenciones no farmacológicas como } \\
\text { movilización temprana, la modulación } \\
\text { ambiental y promoción del sueño antes de } \\
\text { que se utilicen opciones farmacológicas en } \\
\text { un usuario con delirio. }\end{array}$ \\
\hline $\begin{array}{l}\text { E18 Darbyshire } \\
\text { et., al. (2016) }\end{array}$ & $\begin{array}{l}\text { Desarrollar una visión general } \\
\text { amplia de las experiencias de los } \\
\text { pacientes de tratamiento en una } \\
\text { UCI. }\end{array}$ & $\begin{array}{l}\begin{array}{l}\text { Análisis } \\
\text { secundario de } \\
\text { entrevistas } \\
\text { originales }\end{array} \\
\begin{array}{l}77 \text { entrevistas } \\
\text { usuarios, } \\
\text { familiares) }\end{array}\end{array}$ & $\begin{array}{l}\text { Los usuarios y familia, están preocupados por } \\
\text { su estado mental. Los miembros del equipo } \\
\text { de la UCI deben desarrollar estrategias de } \\
\text { afrontamiento como la movilización } \\
\text { temprana, una estructura diaria claramente } \\
\text { definida, orientación repetida y reducción del } \\
\text { ruido, demostrándose con ello, la } \\
\text { disminución de la incidencia del delirio } \\
\text { dentro y fuera de la UCI. }\end{array}$ \\
\hline $\begin{array}{l}\text { E19 Kanji et., al. } \\
\text { (2016) }\end{array}$ & $\begin{array}{l}\text { Investigar validez de la } \\
\text { Herramienta de evaluación del } \\
\text { dolor en pacientes con evidencia de } \\
\text { delirio. }\end{array}$ & $\begin{array}{l}\text { Estudio } \\
\text { prospectivo } \\
\text { cohortes. }\end{array}$ & $\begin{array}{l}\text { Herramienta de observación del dolor de } \\
\text { cuidados intensivos es una herramienta } \\
\text { válida y confiable para detección del dolor en } \\
\text { pacientes adultos de la UCI delirantes no } \\
\text { comatosos. }\end{array}$ \\
\hline
\end{tabular}

Fuente: Elaboración propia 
afectar al usuario desde la unidad de emergencias (Carrasco y Zalaquett, 2017).

\section{RESULTADOS E INTERPRETACIÓN}

A partir del análisis de los documentos seleccionados, emergen cuatro factores que se relacionan con el desarrollo, manejo y evaluación objetiva del delirio, como son: a) La fisiopatología del delirio/factores de riesgos; b) Promoción y prevención para un cuidado integral; c) evaluación objetiva; y d) manejo del delirio en Unidades Críticas.

a) Fisiopatología del delirio/ Factores de riesgo del delirio: De manera general, los expertos afirman que el Delirio, representa la disfunción neurológica más frecuente en pacientes internados en las unidades de terapia intensiva (Da Silva et al., 2017), está presente casi en el $50 \%$ de los pacientes ventilados de forma espontánea (Thomason et al., 2005; Salluh et al., 2010;) y hasta el $80 \%$ de los pacientes ventilados mecánicamente (Pandharipande, 2007; Girald, 2008;). De manera específica, la fisiopatología de las personas deliriosas con enfermedades críticas, aún no se conoce bien (Pisani et al., 2009; Shehabi et al., 2010), pero la etiología parece ser multifactorial y estudios previos han sugerido que el delirio está asociado principalmente con el grado de falla multiorgánica, citosinas inflamatorias, daño microvascular, trombosis, alteración del metabolismo oxidativo e inflamación sistémica (Vitkovic et al., 2000; Girald, 2008; Gofton, 2012; Hughes et al., 2013). Otros estudios neuropatológicos, sugieren que la isquemia neuronal difusa es común en pacientes críticamente enfermos, afectando principalmente las áreas del cerebro susceptibles a lesión hipóxica-isquémica, como las cuencas en la corteza frontal (Sharchar et al., 2004), por lo tanto, la mala perfusión cerebral puede contribuir al delirio (Wood et al., 2017), situación que puede
Respecto a los factores de riesgo, se destaca: Edad avanzada, tabaquismo (Yang et al., 2017), alcoholismo, que si bien la asociación aún no es clara, puede extenderse más allá de la abstinencia alcoholica, puede estar relacionado a deficiencias nutricionales o tener un marcador desconocido para el desarrollo del delirio (Wood et al., 2017), actividad física baja, bajo índice de masa corporal $\quad(<18,6 \mathrm{~kg} / \mathrm{m} 2)$, discapacidad auditiva, fibrilación auricular, antecedentes de accidente cerebrovascular, insuficiencia renal crónica, trastornos del sueño, depresión, antecedentes de delirio, medicación con benzodiazepinas, medicamentos neurolépticos, analgésicos opioides y múltiples medicamentos, polifarmacia, la cual se asocia con aumento de los eventos adversos, mayor interacción de los medicamentos y aumento de los costos (Garpestad y Devlin, 2017), la infección al torrente sanguíneo, inserción de catéteres y drenajes (Dittrich et al., 2016) y finalmente, el adormecimiento o somnolencia identificado con un RASS -1 (Boettger et al, 2017). Por otra parte, los factores precipitantes del delirio fueron la cirugía de emergencia, cirugía abierta, los catéteres permanentes múltiples, uso de analgésicos opioides y benzodiazepinas, la admisión a la unidad de cuidados intensivos (UCI), el ingreso a la sala de emergencias (Kim et al., 2016; Mori et al., 2016), puntaje SOFA ( $\geq 14$ ) (Yang et al., 2017), y el delirio hiperactivo genera estancias más cortas y una menor mortalidad hospitalaria. (Robinson et al., 2011; Swan, 2014).

b) Prevención y promoción del delirio para un cuidado integral: Dentro de las medidas preventivas para el delirio, se destacan la reducción de los factores de riesgos (mencionados en el punto anterior), y, por otra parte, se reconocen algunas acciones destinadas a detener su avance y atenuar sus consecuencias una vez establecida. Según 
Revista científica de la Asociación de Historia y Antropología de los Cuidados (Universidad de Alicante)

ésta afirmación, y basados, en los artículos académicos revisados, se sugieren las siguientes acciones:

- Disponer un modelo predictivo del delirio en las UCI, lo que permitirá identificar a los usuarios de alto riesgo, para monitoreo e implementación proactiva de estrategias preventivas (Kim et al., 2016).

- Contar con herramientas de evaluación de delirio, así se podrá identificar a usuarios con mayor predisposición al delirio y, además, se podrían optimizar los recursos (Kim et al., 2016).

- Potenciar la "gestión del riesgo" en la UCI, puntualmente por parte de los enfermeros especialistas, ya que ellos tienen las competencias en el ámbito de la creación y el mantenimiento de entornos terapéuticos seguros (Pereira et al., 2017).

- Optimizar el manejo del dolor y la sedación (Reade, Finfer, 2014).

- Contar con un modelo de visita extendida, ya que acorta la duración del delirio, disminuye el estrés y la ansiedad (Gourlart et al., 2017).

- Implementar un protocolo de delirio con intervenciones farmacológicas y no farmacológicas (Sullinger et al., 2016). Sin duda alguna, uno de los aspectos más importantes es el tratamiento farmacológico, y si bien, es parte del rol médico, enfermería sugiere $\mathrm{y}$ participa activamente en la administración el tratamiento (Morandi et al., 2011), y debe conocer cuáles son los medicamentos que puedan causar el delirio, ya coordinar modificaciones (Oto et al., 2011).

- Utilizar el Bundle ABCDEF de cuidados críticos que integra: la evaluación, prevención y manejo del dolor, despertar y respirar espontáneas, analgesia, sedación, delirio, movilidad temprana y participación de la familia. (Morandi et al., 2017; Sullinger et al., 2016).
Es necesario fortalecer la promoción de salud a todo nivel, la cual se puede realizar a través de la educación para el usuario y la familia en temas relacionados a:

- Estrategias de afrontamiento como la movilización temprana, la orientación repetida y la reducción del ruido (Darbyshire et al., 2016)

- Necesidad de educar a estos profesionales respecto a la evaluación rutinaria y tratamiento oportuno del delirio, ya que solo el $31 \%$ de lo que evaluaron el delirio utilizaron una herramienta validada. (Xing et al., 2017)

De tal manera para poder promover y prevenir los factores que, relacionados al delirio en los usuarios hospitalizados en UCI, ser debe saber sobre la gestión del cuidado, ya que ésta es una labor rigurosa que requiere de análisis, deducción, discernimiento y conocimiento; es decir, la aplicación de "juicio enfermero", sustentado en el ser, saber y quehacer de la Enfermería como profesión y disciplina. (CeballosVásquez et al., 2015).

c) Evaluación objetiva del delirio

Existen escalas para evaluar el delirio, entre las cuales se destacan: Confusión Assessment Method for the Intensive Care Unit (CAM-ICU); Intensive Care Delirium Screening Checklist (ICDSC); Edinburgh Delirium Test Box-ICU (EDTB-ICU); Nursing delirium screening checklist (NuDESC); CAM-ICU 7; Delirium Rating Scale-Revised-98 (DRS-R-98); Cognitive Test for Delirium; Neecham Scale y Delirium Detection Score. Sin embargo, las guías 2013 de la Sociedad de Medicina de Cuidados Críticos (SCCM), recomiendan el uso del método de evaluación de confusiones para la UCI (CAM-ICU) o la lista de verificación de detección de delirium de cuidados intensivos (ICDSC), ya que las reconoce como herramientas validadas y confiables en la UCI (Barr et al., 2013). Por 
lo señalado, se sugiere la incorporación de CAM-ICU en la valoración de enfermería para usuarios críticos (Barr et al., 2013). Se evidencia también que los encargados de objetivar el delirio son los enfermeros y/o médicos, debido a que son los que deben realizar una valoración diaria del usuario. Los artículos recomiendan contar una herramienta de evaluación para el delirio, que sea objetiva y validada, y debería ser aplicada al menos una vez por turno o cada 12 horas (Pereira et al., 2016; Da Silva et al., 2017).

d) Manejo del delirio en Unidades Críticas

e)

Respecto al manejo, los artículos recomiendan primeramente contar un equipo capacitado, reconociendo de manera precoz esta alteración, entregando un manejo y tratamiento oportuno (Xing et al., 2017). Utilizar una herramienta de evaluación, contar con un protocolo para el manejo del delirio que entregue indicaciones que sean conocidas por todo el personal y de esta forma lograr estandarizar los cuidados (Sullinger et al., 2016). El uso de los BUNDLE ABCDEF para Cuidados Críticos ayudaría a tener un mejor manejo (Morandi et al., 2017). Y, por último, tener en cuenta todos los factores de riesgo que puedan propiciar el desarrollo del delirio, incluyendo el manejo de la oxigenación, por sobre todo en las primeras 24 horas, ya que son las horas claves de esta alteración (Wood et al., 2017).

\section{CONCLUSIONES}

Aunque se estudia hace algunas décadas, aún no se conoce de manera clara, su etiología. Si bien, se reconocen muchos factores que influyen para su desarrollo, como la edad avanzada, polifarmacia, multiplicidad de elementos invasivos, uso de sedantes entre otros. Aún existe un desconocimiento por parte del equipo de salud en la identificación del delirio, en su promoción, prevención y manejo de éste. A pesar de ello, existen herramientas validadas para evaluarlo. Enfermería es fundamental en la pesquisa y el actuar precoz, puesto que es el profesional de salud que pasa la mayor parte del tiempo al cuidado del paciente, conociéndolo desde su ingreso y durante toda su evolución intrahospitalaria hasta el alta.

La Gestión del Cuidado en la legislación chilena, es rol de enfermería, por ende, es fundamental relevar esta temática, debido a que su incidencia aumentará los días de hospitalización, días de ventilación mecánica y mortalidad sólo por no encontrarse familiarizados con un tema tan recurrente en UCI. Finalmente sería interesante seguir investigando en Chile y el mundo, sobre el uso de una herramienta objetiva para evaluar el delirio en el usuario crítico e instalar un protocolo de manejo.

\section{BIBLIOGRAFÍA}

Barr, J., Fraser, GL., Puntillo, K., Ely, EW., Gélinas, C., Dasta, JF., Davidson, JE., Devlin, JW..., y Jaeschke, R. (2013). Clinical Practice Guidelines for the Management of Pain, Agitation, and Delirium in Adult Patients in the Intensive Care Unit. Critical Care Medicine. 41(1), 263-306.

Boettger, S., Nuñez, D., Meyer, R., Richter, A, Fernández, S., Rudiger, A., Schubert, M., y Jenewein, J. (2017). Delirium in the intensive care setting and the Richmond Agitation and Sedation Scale (RASS): Drowsiness increases the risk and is subthreshold for delirium. Journal of Psychosomatic Research, 103,133-39.

Bui, L., Pham, V., Shirkey, B., y Swan, J. (2017). Effect of delirium motoric subtypes on administrative documentation of delirium in the surgical intensive care 
Revista científica de la Asociación de Historia y Antropología de los Cuidados (Universidad de Alicante)

unit. Journal of clinical monitoring and computing, 31, 631-40.

Carrasco, M., y Zalaquett, M. (2017). Delirium: una epidemia desde el servicio de urgencia a la unidad de paciente crítico. Revista Médica de Clínica las Condes, 28 (2), 301-10.

Ceballos, P., Jara, A., Stiepovich, J. Aguilera, P. y Vílchez, V. (2015). La gestión del cuidado: una función social y legal de la enfermería chilena. Revista de Enfermería Actual en Costa Rica, 29, 1-12. doi: http://dx.doi.org/10.15517/revenf.v0i29.197 33.

Da Silva, R., Ribeiro, M., Pires, E., Meira, L., Lepre, D., y De Oliveira, N. (2017). Nurses' training in the use of a delirium-screening tool. Revista Gaúcha de Enfermagem, 38 (1). doi: 10.1590/19831447.2017.01.64484.

Darbyshire, J., Greig, P., Vollam, S., Young, D., y Hinton, L. (2016). I Can Remember Sort of Vivid People . . but to Me They Were Plasticine. Delusions on the Intensive Care Unit: What Do Patients Think Is Going On? PLOS ONE 11 (4). doi:10.1371/journal.pone.0153775.

Dittrich, T., Tschudin-Sutter, S., Widmer, A., Rúegg, S., Marsch, S., y Sutter, R. (2016). Risk factors for new-onset delirium in patients with bloodstream infections: independent and quantitative effect of catheters and drainages- a fouryear cohort study. Annals of intensive care, $6(1), 104$.

Ely, E., Shintani, A., Truman, B., Speroff, T., Gordon, S., Harrell, F., Inouye, S... y Dittus, R. (2004). Delirium as a Predictor of Mortality in Mechanically Ventilated Patients in the Intensive Care Unit. Journal of the American Medical Association. 291(14), 1753-62.
Garpestad, E., y Devlin, J. W. (2017). Polypharmacy and Delirium in Critically Ill Older Adults. Clinics in Geriatric Medicine, 33(2), 189-203.

Girard, T., Kress, J., Fuchs, B., Thomason, J., Schweickert, W., Pun, B., Taichman, D.....y Ely, E. (2008). Efficacy and safety of a paired sedation and ventilator weaning protocol for mechanically ventilated patients in intensive care (Awakening and Breathing Controlled trial): a randomized controlled trial. The Lancet, 371(9607), 126-34.

Girard, T., Ware, L., Bernard, G., Pandharipande, P., Thompson, J., Shintani, A., Jackson, J.C.., y Ely, E. (2012). Associations of markers of inflammation and coagulation with delirium during critical illness. Intensive Care Medicine, 38(12), 1965-1973.

Gofton, T., y Young, G. (2012). Sepsisassociated encephalopathy. Nature Reviews Neurology, 8:557. http://dx.doi.org/10.1038/nrneurol.2012.183

Goulart, R., Tonietto, T., Barbosa, D., Gutierres, F., Ascoli, A., Cordeiro, L... y Teixeira, C. (2017). Effectiveness and Safety of an Extended ICU Visitation Model for Delirium Prevention: A Before and After Study. Critical Care Medicine, 45 (10), 1660-7.

Green, C., Hendry, K., Wilson, E., Walsh, T., Allerhand, M., MacLullich, A., y Tieges, Z. (2017). A Novel computerized Test for Detecting and Monitoring Visual Attentional Deficits and Delirium in the ICU. Critical Care Medicine, 45(7), 122431.

Hughes, C., Morandi, A., Girard, T., Riedel, B., Thompson, J., Shintani, A., Pun..., y Pandharipande, P. (2013). Association between Endothelial Dysfunction and Acute Brain Dysfunction 
during Critical Illness. Anesthesiology, 118(3), 631-39.

Kanji, S., MacPhee, H., Singh, A., Johanson, C., Fairbairn, J., Lloyd, T., MacLean, R. y Rosenberg, E. (2016). Validation of the Critical Care Pain Observation Tool in Critically Ill Patients with Delirium: A Prospective Cohort Study. Critical Care Medicine, 44(5), 943-7.

Khan, B., Perkins, A., Gao, S., Hui, S., Campbell, N., Farber, M.,... y Boustani, M. (2017). The Confusion Assessment Method for the ICU-7 Delirium Severity Scale: A Novel Delirium Severity Instrument for Use in the ICU. Critical Care Medicine, 45(5), 851-7.

Kim, M., Park, U., Kim, H., y Cho, W. (2016). Delirium Prediction Based on Hospital Information (Delphi) in General Surgery Patients. Medicine, 95 (12).

Morandi, A., Piva, S., Wesley, E., Myatra, S., Salluh, J., Amare, D., Azoulay, E., Bellelli..., y Latronico, N. (2017). Worlwide Survey of the "Assessing Pain, Both Spontaneous Awakening and Breathing Trials, Choice of Drugs, Delirium Monitoring/ Management, Early Exercise/ Mobility, and Family Empowerment" (ABCDEF) Bundle. Critical Care Medicine, 20 (30).

Morandi, A., Vasilevskis, EE., Pandharipande, PP., Girard, TD., Solberg, LM., Neal, EB., Koestner, T..., y Kripalani, S. (2011). Inappropriate medications in elderly ICU survivors: where to intervene? Archives of Internal Medicine, 171(11):1032-4.

Mori, S., Tsuchihashi Takeda, JR., Angotti Carrara, FS., Rizzo Cohrs, C., Viski Zanei, SS., y Yamaguchi Whitaker, I. (2016). Revista da Escola de Enfermagem USP, 50(4), pp. 585-91.

Oto, J., Yamamoto, K., Koike, S., Imanaka, H., y Nishimura, M. (2011). Effect of daily sedative interruption on sleep stages of mechanically ventilated patients receiving midazolam by infusion. Anaesthesia Intensive Care, 39, 392-400.

Pandharipande, P., Pun, B., Herr, D., Maze, M., Girard, T., Miller, R., Shintani, A..., Ely, E. (2007). Effect of sedation with dexmedetomidine vs lorazepam on acute brain dysfunction in mechanically ventilated patients: the MENDS randomized controlled trial. JAMA, 298(22), 2644-53.

Palencia, E., Romera, M., Silva, J., y grupo de trabajo de analgesia y sedación de la Semicyuc. (2008). Delirio en el paciente crítico. Medicina Intensiva, 32(1), 77-91.

Pereira, J., Dos Reis, F., Caetano, R., Pinto, M., Batista, M., Galhardas, M., y Santos, M. (2016). Delírium no doente crítico: fatores de risco modificáveis pelos enfermeiros. Revista de Enfermagen Referencia, 4(9),29-36.

Pisani, M. A., Kong, S. Y. J., Kasl, S. V., Murphy, T. E., Araujo, K. L. B., y Van Ness, P. H. (2009). Days of Delirium Are Associated with 1-Year Mortality in an Older Intensive Care Unit Population. American Journal of Respiratory and Critical Care Medicine, 180(11), 1092-97.

Reade, M., Phil, D., y Finfer, S. (2014). Sedation and Delirium in the Intensive Care Unit. The New England Journal of Medicine. 370, 444-54.

Robinson, T., Raeburn, C., Tran, Z., Brenner, L., y Moss, M. (2011). Motor subtypes of postoperative delirium in older adults. The Archives of Surgery, 146(3), 295-300.

Salazar, N., Griñen, H., Jirón, M., Rojas, L., Escobar, L., Berasaín, M., y Romero, C. (2012). Impacto del cuidado multidisciplinario en los desenlaces clínicos de los pacientes Críticos. Revista Chilena de Medicina Intensiva, 27(1), 12-22. 
Revista científica de la Asociación de Historia y Antropología de los Cuidados (Universidad de Alicante)

Salluh, J.I., Soares, M., Teles, J.M., Ceraso, D., Raimondi, N., Nava, V.S., Blasquez, P., Ugarte, S..., y Rocha, M.G., Delirium Epidemiology in Critical Care Study Group. (2010). Delirium epidemiology in critical care (DECCA): an international study. Crit Care, 14(6), 210.

Salluh, J., Wang, H., Schneider, E., Nagaraja, N., Yenokyan, G., Damluji, A., Serafim, R., y Stevens, R. (2015). Outcome of delirium in critically ill patients: systematic review and meta-analysis. British medical journal. 350, h2538.

Severgnini, C., Dornelles, A., Bessel, M., Meira, J., Skrobik, Y., y Teixeira, C. (2017). Effectiveness and Safety of an Extended ICU Visitation Model for Delirium Prevention: A Before and After Study. Critical Care Medicine, 45(10), 1660-67.

Sharshar, T., Annane, D., de la Grandmaison, GL., Brouland, JP., Hopkinson, NS., y Françoise, G. (2004). The neuropathology of septic shock. Brain Pathology, 14(1): 21-33.

Shehabi, Y., Riker, R.R., Bokesch, P.M., Wisemandle, W., Shintani, A., y Ely, W. SEDCOM (Safety and Efficacy of Dexmedetomidine Compared With Midazolam) Study Group. (2010). Delirium duration and mortality in lightly sedated, mechanically ventilated intensive care patients. Critical Care Medicine, 38(12), 2311-18.

Sullinger, D., Gilmer, A., Jurado, L., Hall, L., Steelman, J., Gallagher, A., Dupre, T., y Acquista, E. (2016). Development, Implementation, and Outcome of a Delirium protocol in the Surgical Trauma Intensive Care Unit. Annals of Pharmacotherapy, 51(1), 5-12.

Swan, J. (2014). Decreasing inappropriate unable-to-assess ratings for the confusion assessment method for the intensive care unit. AJCC, 23(1):60-9.

Thomason, J., Shintani, A., Peterson, J., Pun, B., Jackson, J., y Ely, E. (2005). Intensive care unit delirium is an independent predictor of longer hospital stay: a prospective analysis of 261 nonventilated patients. Critical Care, 9(4), 375.

Van den Boogaard, M., Schoonhoven L., Evers, A., Van der Hoeven, J., Van Achterberg, T., y Pickkers, P. (2012). Delirium in critically ill patients. Critical Care Medicine, 40 (1), 112-118.

Vitkovic, L., Konsman, JP, Bockaert, J., Dantzer, R., Homburger, V., y Jacque, C (2000).Cytokine signals propagate through the brain. Molecular Psychiatry, 5(6), 60415.

Wood, M., Maslove, D., Muscedere, J., Day, A., y Gordon, J. (2017). Low brain tissue oxygenation contributes to the development of delirium in critically ill patients: A prospective observational study. Journal of Critical Care, 41, 289-95.

Wolters, A., Peelen, L., Welling, M., Kok, L., de Lange, D., Cremer, O., Van Dijk, D..., y Veldhuijzen, D. (2016). Long- Term Mental Health Problems after Delirium in the ICU. Critical Care Medicine, 44 (10), 1808-13.

Xing, J., Sun, Y., Jie, Y., Yuan, Z., y Liu, W. (2017). Perceptions, attitudes, and current practices regards delirium in China. Medicine, 96:39.

Yang, J., Zhou, Y., Kang, Y., Xu, B., Wang, P., Lv Y., y Wang Z. (2017). Risk Factors of Delirium in Sequential Sedation Patients in Intensive Care Units. BioMed Research International 3,1-9. doi: 10.1155/2017/3539872Hindawi, 2017. 